\title{
Are There Indications for Total Pancreatectomy in 2016?
}

\author{
Åke Andrén-Sandberg ${ }^{\mathrm{a}}$ Christoph Ansorge ${ }^{\mathrm{a}}$ Thakur Deen Yadav ${ }^{\mathrm{b}}$ \\ ${ }^{a}$ Department of Digestive Diseases, Karolinska University Hospital, Stockholm, Sweden; ${ }^{\mathrm{b}}$ Department of General \\ Surgery PGIMER, Chandigarh, India
}

\section{Key Words}

Total pancreatectomy $\cdot$ Pancreatic surgery $\cdot$ Pancreatic resection

\begin{abstract}
An elective total pancreatectomy (TP) was first performed by Eugene Rockey of Portland, Oregon, in 1942. In the 1960s and 1970s, TP was the routine resection for pancreatic cancer in many centers because of fear of a leaking pancreatojejunostomy and multicentricity of the disease but the result used to be dreadful (in today's perspective). However, more recently, postoperative mortality and morbidity after pancreatic resections have improved due to better anastomotic technique and pre-, peri- and postoperative care. Today, TP despite being a more extensive operation - can be offered with about the same operation risk as that of a Whipple procedure. Also, major improvements in the control of diabetes have been seen and there is actually an ongoing discussion on the actual severity of the diabetic state after TP. Also, the development of modern pancreatic enzyme preparations with sufficient control of endocrine and exocrine pancreatic insufficiency provides options for overcoming the postoperative problems following TP. Due to the improved results, there are today different - and more specific - indications than before for TP: malignant tumors growing from the pan-
\end{abstract}

creatic head into the left pancreas, pancreatic head cancer where it is not possible to secure a tumor-free resection margin with extended resection or with dubious changes in the pancreatic main duct at frozen section, recurrent malignancy in the pancreatic remnant, at cancer surgery with resection of the celiac trunk, rescue pancreatectomy after a leaking pancreatojejunostomy with sepsis or bleeding after a Whipple-type first resection, multifocal intraductal papillary mucinous neoplasm with potentially malignant foci present in all parts of the gland, multiple metastases of renal cell carcinoma and melanoma without any residual tumor outside the pancreatic gland (possibly also other specified but uncommon metastatic tumors with a potential for cure by pancreatectomy), multifocal neuroendocrine tumors including multiple endocrine neoplasia and hereditary pancreatic cancer with a high grade of cancer penetration risk for the bearers.

(c) 2016 S. Karger AG, Basel

\section{Introduction}

Postoperative complications after pancreatic resections are mainly related to problems with the pancreatic anastomosis. Therefore, various techniques have been developed to deal with the pancreatic stump. One of

\section{KARGER}

E-Mail karger@karger.com www.karger.com/dsu
(C) 2016 S. Karger AG, Basel

0253-4886/16/0334-0329\$39.50/0
Åke Andrén-Sandberg

K53, Karolinska University Hospital

14186 Stockholm (Sweden)

E-Mail ake.andrensandberg@gmail.com 
them is - or was - total pancreatectomy (TP) [1]. Besides avoiding a pancreatointestinal anastomosis, TP was also considered as an extension of oncological radicality in pancreatic cancer patients. High local recurrence rates after cranial pancreatoduodenectomy ('Whipple resection') [2] and left-sided pancreatectomy ('tail resection') suggested once that pancreatic cancer might develop multicentrically in the pancreatic gland, which made TP a logical choice. The indications and enthusiasm for the TP has changed significantly. A further indication for TP is the situation where it is needed in the emergency setting as salvage procedure to control sepsis. The earlier indications were based on the premise of multifocality of the adenocarcinoma of the pancreas and it was presumed that TP would be required have more oncological clearance. However, in practice, the outcome of TP to get more oncological clearance and to decrease the complications of partial pancreatectomy has been disappointing.

More recently, the indication has taken a significant change due to better understanding of the biology of tumor and evolution in intervention technique and better postoperative care. Now, the alternative indications are intraductal papillary neoplasms (IPMN), neuroendocrine tumors and secondaries from renal cell carcinoma chronic pancreatitis in the setting of intractable pain. TP may also be needed to have free resection (R0) margin while performing partial pancreatectomy.

\section{Historical Notes}

Although TP was performed by Minkowski [3] in the dog in 1898 in Breslau, Germany, the procedure could not be performed successfully in humans before the discovery of insulin in 1921. Several reports suggest that Theodor Billroth from Vienna undertook the first total or near-TP in humans in 1884 with good outcome (anecdotal report by Arthur William Mayo-Robson in a speech given to an international medical congress in Paris in 1901) [4]. An elective, 'true', TP appears first to have been performed by Eugene Rockey of Portland, Oregon, in 1942, but the patient died 15 days later of bile peritonitis due to leakage from the common bile duct [3]. Three weeks later, James Priestly of Rochester, Minnesota, performed TP on a 40-year-old woman who suffered from repeated severe hypoglycemic episodes. Priestly could not find the pancreatic tumor on exploration and therefore made the radical decision to perform a TP for what turned out to be a $1 \mathrm{~cm}$ insulinoma [5]. The patient survived for 29 years before dying of cholangitis. In 1948, Gaston [6] gathered a report of 17 cases from the scientific literature and published it.

\section{Benefits vs. Disadvantages}

After the first enthusiasm over the ability to perform $\mathrm{TP}$, the disadvantages of this surgical procedure became more obvious. Several centers reported perioperative mortality and morbidity rates equal to those of the Whipple procedure, but without improvements in the longterm survival. In admission, TP always resulted in major metabolic problems such as brittle insulin-dependent diabetes mellitus, which used to be difficult to control. Moreover, malabsorption due to loss of exocrine pancreatic secretion was also often difficult to manage. Therefore, weight loss, diarrhea and malabsorption often contributed to a cachexia-like syndrome that significantly decreased the quality of life and the possibility of normal physical activity. Steatorrhea and the short food transit time resulting in inestimable carbohydrate absorption, which in turn made it difficult to titrate the insulin dose to the food intake. The steatorrhea also contributed to the loss of fat-soluble vitamins, at least vitamin $\mathrm{D}$, leading to osteopathy and osteoporosis as well as liver disorders. Repeated attacks of hypoglycemia and hypoglycemic coma used to occur to initial survivor.

Due to the problems of exocrine and endocrine insufficiency and the reduced quality of life, without oncological benefit, TP was at large abandoned in clinical practice and no longer considered to be a viable option for treatment of pancreatic tumors for many years [1].

There are several recent studies that have shown that initial morbidity and mortality of TP has been almost equivalent to that of the partial pancreatectomy performed in the same period of time. Müller et al. [1], in a series of 147 patients of TP done for elective and emergency indications, have shown that perioperative mortality and morbidity for elective TP have been quite acceptable (mortality $4.8 \%$ and total morbidity $36 \%$ ), and they could not find any statistical difference with when compared to the partial pancreatectomy done in the same period. Almost same type of the result has been very recently published by Epelboym et al. [7] who in a retrospective study of 77 patients operated for different indications have shown that perioperative mortality and morbidity is almost equivalent to the partial pancreatectomy done during the same period. 


\section{Pancreatic Malabsorption}

Therapy for pancreatic exocrine insufficiency is based on oral administration of pancreatic enzymes, aiming at providing the duodenal lumen with sufficient lipase at the time of gastric emptying. Administration of enzymes in the form of enteric-coated minimicrospheres avoids acidmediated lipase inactivation and ensures gastric emptying of enzymes in parallel with nutrients. Nevertheless, factors such as acidic $\mathrm{pH}$ of the stomach and bacterial overgrowth may be taken into account to normalize fat digestion even in complicated patients. However, for various reasons, impairment of pancreatic lipase activity cannot be sufficiently compensated by other mechanisms. Digestion of fats in the small intestine is mainly due to the combined effects of lipase and its cofactors, especially bile acids. Intestinal mucosa does not express enzymatic systems that digest triglycerides, and lipolytic activity produced by other extrapancreatic sources (salivary and gastric) cannot compensate lipid digestion by pancreatic lipase [8]. Despite that, current therapeutic approaches to pancreatic exocrine insufficiency make it possible to eradicate steatorrhea in almost all cases [9]. Barbier et al. [10] have published short- and long-term impact of TP in series of 56 patients. Of the 30 surviving patients 15 lost weight, with a median of $9 \mathrm{~kg}(2-14)$, and their weight at the time of analysis was $85 \%$ (78-97) of their preoperative weight. Four patients gained weight (13 kg, range 4-22). The 25 patients had $2(1-5)$ stools per day, which were liquid in one-quarter of the patients. Seven patients had night stools, and for 5 patients, diarrhea stools were a limiting factor in everyday life. The median daily intake of pancreatic enzymes supplements was 6 (318 ) capsules $(25,000$ units per capsule) and 5 patients took other medications for intestinal transit, including loperamide $(\mathrm{n}=1)$ and diosmectite $(\mathrm{n}=1)$. Liver function test and prothrombin time were within normal ranges; particularly, no cholestasis was noticed. The renal function was usually preserved with a median serum creatinine of $82 \mathrm{mmol} / \mathrm{l}$ (50-163). Nutrition markers included albumin of $100 \%(81-100)$, pre-albumin $100 \%(70-100)$ and orosomucoid $100 \%(63-100)$ of normal values.

\section{Pancreatic Diabetes}

Pancreatogenic diabetes mellitus (T3cDM) after pancreatic resection differs from types 1 and 2 diabetes. Unlike type 1, pancreatogenic diabetes seldom causes ketoacidosis or severe hyperglycemia. Pancreatogenic diabetes is also unlike type 2 diabetes, which is characterized by insulin resistance and relative insulin deficiency. Pancreatogenic diabetes is highly sensitive to insulin. The pan-

Are There Indications for TP in $2016 ?$ creatogenic diabetes is attracting attention as the primary factor influencing quality of life in patients who have undergone this procedure, with the difficulty to predict the effect on carbohydrate metabolism because of metabolic changes. Experimental results suggest that insulin-independent glucose metabolism after pancreatic resection is due to a large proportion of absorbed glucose. This may explain why patients with pancreatogenic diabetes behave differently than other diabetics sensitive to insulin therapy. These patients with unmanageable diabetes 'brittle diabetics' - may become unpredictable hypoglycemics during treatment with insulin, unrelated to food intake or physical effort. The nutritional status, pancreatic exocrine function and intestinal function also affect the glycemic control. These patients have an increased serum level of gluconeogenetic precursors such as lactate and alanine [9]. These distinct clinical and metabolic aspects have been attributed to pancreatic glucagon secretion deficits [10]. Pancreatogenic diabetics have elevated levels of serum insulin and minimal or absent response to food intake, as opposed to a type 1 diabetics, where serum insulin is normal or elevated and there is an exaggerated response to ingestion of sugar [11].

Because of the increased peripheral sensitivity to insulin and the reduced glucagon level in pancreatogenic diabetes, exogenous insulin administration frequently even causes hypoglycemic attacks. Iatrogenic hypoglycemia may occasionally lead to hospitalization, irreversible damage to the central nervous system, or even death [12, 13]. However, with increasing knowledge of pathophysiology of T3cDM, both the short-and long-term results now are good and long-term survival is dependent almost only on the indications for pancreatic resection [12]. Epelboym et al. [7] in their recently published study on the quality of life after TP have shown a negative impact of diabetes assessed by audit of diabetes-dependent quality of life of the patients. However, they could not find any differences between total pancreatectomy and partial pancreatectomy in 'life domains'. The most negatively impacted by diabetes involved travel and physical activity, whereas self-confidence, friendships and personal relationships, motivation and feelings about the future remained unaffected.

\section{New Indications}

In the 1960s and 1970s, TP was the routine resection for pancreatic cancer in many centers, at least in Scandinavia. There were 2 reasons behind this - the fear of a 
leaking pancreatojejunostomy and multicentricity of the disease - as a consequence, the result used to be dreadful (in today's perspective) [12]. However, more recently, postoperative mortality and morbidity after all pancreatic resections have, due to better anastomotic technique, better pre-, peri- and postoperative care. Today TP - despite being a more extensive operation - can be offered with about the same operation risk as that of a Whipple procedure. Also, major improvements in the control of diabetes have been seen, and there is actually an ongoing discussion on the actual severity of the diabetic state after TP. Several groups have reported equivalent HbAlc levels between type 1 diabetics and pancreatectomized patients [1, 14-16]. Also, the development of modern pancreatic enzyme preparations with sufficient control of endocrine and exocrine pancreatic insufficiency provides options for overcoming the postoperative problems following TP $[1,17,18]$.

Due to the improved results, there are today different - and more specific - indications than before for TP. Even in cases of adenocarcinoma, the understanding of the tumor biology and the pathophysiology of the complications are much clearer than ever.

- Malignant tumors growing from the pancreatic head into the left pancreas;

- Pancreatic head cancer where it is not possible to secure a tumor-free resection margin with extended resection or with dubious changes in the pancreatic main duct at frozen section;

- Recurrent malignancy in the pancreatic remnant;

- At cancer surgery with resection of the celiac trunk;

- Rescue pancreatectomy after a leaking pancreatojejunostomy with sepsis or bleeding after a Whipple-type first resection;

- Multifocal IPMN with potentially malignant foci present in all part of the gland;

- Multiple metastases of renal cell carcinoma and melanoma without any residual tumor outside the pancreatic gland (possibly also other specified but uncommon metastatic tumors with a potential for cure by pancreatectomy);

- Multifocal neuroendocrine tumours including multiple endocrine neoplasia;

- Hereditary pancreatic cancer with a high grade of cancer penetration risk for the bearers.

Despite the rather long list of indications, TP is still not common in the practice of pancreatic cancer surgery. It comprises about $10 \%$ of all resections in recent larger series [17-19]. Moreover, TP remains a viable option in the treatment of intractable pain associated with chronic pancreatitis, especially in patients who do not abuse alcohol or tobacco and where it is possible to autotransplant the islets of Langerhans [20-22].

\section{Intraductal Papillary Mucinous Neoplasm}

Quantitatively, the most important indication today seems to be for IPMN. International consensus guidelines for the management of IPMN recommend resection for main duct disease with clearance of all highgrade dysplasia (HGD) $[23,24]$. As main duct IPMN may be associated with extensive multifocal HGD, the role of TP in this setting is increasingly recognized. The decision may be made preoperatively at the multidisciplinary therapy conference or intraoperatively in patients with positive margins on frozen section. Murphy et al. [25] reported increasing nationwide utilization of TP in the US between 1998 and 2006 and concluded that the rise in was a consequence of increased diagnosis of diffuse neoplastic diseases, particularly IPMN $[26,27]$.

\section{Modified Surgical Techniques}

The procedure of TP has usually included distal gastrectomy to prevent stomal ulcers at the gastroenteric anastomosis and splenectomy to prevent ischemia as a result of a decreased blood supply to the spleen (in malignancy the splenic artery and vein are always resected, which means that only the short vessels between spleen and stomach are left intact). However, today there are modifications that might give a better postoperative life for the patient.

\section{Pylorus-Preserving TP}

The role of pylorus-preserving TP (PpTP) has not yet been settled, but has been discussed as an alternative firstly in cases of borderline and malignant IPMN. However, in a non-randomized manner, 15 PpTPs were compared to 33 with 'standard' TP but preservation of the pyloric ring provided little or no benefit to shortterm outcome or long-term nutritional status among patients who underwent TP [28]. Multiple studies have demonstrated that pylorus-preserving pancreaticoduodenectomy (PpPD) has equal or superior outcomes regarding quality of life when compared with the traditional Whipple operation, but many studies have suggested a higher incidence of delayed gastric emptying (DGE). DGE prolongs hospital stay, and its association with PpPD has hampered its adoption by many pancre- 
atic surgery centers. There are still not enough data to tell if PpTP offers any advantages compared to 'standard' pancreatectomy regarding oncological principles as there are no indications that it should be of inferior value [29]. However, the lack of data despite almost 10 years practice of the method speaks in favor of little advantages.

\section{Stomach- and Spleen-Preserving TP}

Pylorus- and spleen-preserving total pancreatoduodenectomy (PpSpTPD) with segmental resection of both splenic vessels has also been attempted. The technique is based on the concepts of 2 surgical procedures: PpPD and distal pancreatectomy with segmental resection of splenic vessels ('extended' Warshaw's procedure). In one small series, 5 patients underwent laparoscopic-assisted PpSpTPD and 2 underwent open surgery. No mortality was noted. Short-term follow-up (median 28 months) suggested that all patients tolerated the insulin therapy and showed relatively good nutritional status. Only minimal to moderate perigastric fundal varices were noted without gastrointestinal bleeding. Thus, PpSpTPD with segmental resection of both splenic vessels can be indicated in selected patients [30]. The procedure has also been performed laparoscopically [31].

\section{Future Prospects}

There is a clear general trend in gastrointestinal surgery toward minimally invasive surgery, which means that TP - and extended pancreatectomies as described and made popular for a while by Fortner [32] - ought to be thought of as yesterday's surgical strategies. However, not least due to the interest in preventing pancreatic cancer by following up IPMNs and other cystic tumors, it is probable that we will see more total pancreatectomies in the nearest future. However, it is probable that we will also get better imaging of small pancreatic lesions, thus picking out those cystic - and other pancreatic tumors - that might be malignant. Also, it is most probable that we will learn how to destruct small pancreatic tumors without open surgery (e.g., by radiofrequency ablation) [33] and in a somewhat longer perspective even treat premalignant lesions and turn them into benign lesions by pharmacological means. In this scenario, the number of total pancreatectomies will almost vanish again, except for the advanced pancreatic adenocarcinomas and the rescue pancreatectomies after leaking pancreatoenteric anastomoses. This means that even if the frequency with which we perform TP will vary according to prerequisites, it will still be needed sometimes in our pancreatic surgery arsenal also in the future.

\section{References}

$\checkmark 1$ Müller MW, Friess H, Kleeff J, Dahmen R, Wagner M, Hinz $U$, et al: Is there still a role for total pancreatectomy? Ann Surg 2007;246: 966-974; discussion 974-975.

2 Westerdahl J, Andrén-Sandberg A, Ihse I: Recurrence of exocrine pancreatic cancer - local or hepatic? Hepatogastroenterology 1993;40: 384-387.

3 Minkowski O: De l'exirpation des pancéas chez les animoux et du diabète expérimental. Semaine Med (Paris) 1889;9:175-176.

4 Rockey EW: Total pancreatectomy for carcinoma: case report. Ann Surg 1943;118:603611.

5 Priestley JT, Comfort MW, Radcliffe J: Total pancreatectomy for hyperinsulinism due to an islet-cell adenoma: survival and cure at sixteen months after operation presentation of metabolic studies. Ann Surg 1944;119:211221.

6 Gaston EA: Total pancreatectomy. N Engl J Med 1948;238:345-354.

7 Epelboym I, Winner M, DiNorcia J, et al: Quality of life in patients after total pancreatectomy is comparable with quality of life in patients who undergo a partial pancreatic resection. J Surg Res 2014;187:189196.
-8 Layer P, Holtmann G: Pancreatic enzymes in chronic pancreatitis. Int J Pancreatol 1994;15: $1-11$.

-9 Domínguez-Muñoz JE: Pancreatic enzyme therapy for pancreatic exocrine insufficiency. Curr Gastroenterol Rep 2007;9:116-122.

10 Barbier L, Jamal W, Dokmak S, et al: Impact of total pancreatectomy: short- and long-term assessment. HPB (Oxford) 2013;15:882-892.

11 Karmann H, Laurent F, Mialhe P: Pancreatic hormones disappearance after total pancreatectomy in the duck: correlation between plasma glucagon and glucose. Horm Metab Res 1987;19:538-541.

12 Unger RH: Glucagon physiology and pathophysiology. N Engl J Med 1971;285:443-449.

$\checkmark 13$ Slezak LA, Andersen DK: Pancreatic resection: effects on glucose metabolism. World J Surg 2001;25:452-460.

14 Maeda H, Hanazaki K: Pancreatogenic diabetes after pancreatic resection. Pancreatology 2011;11:268-276.

15 Andrén-Sandberg A, Ihse I: Factors influencing survival after total pancreatectomy in patients with pancreatic cancer. Ann Surg 1983; 198:605-610.

16 Billings BJ, Christein JD, Harmsen WS, Harrington JR, Chari ST, Que FG, Farnell MB,
Nagorney DM, Sarr MG: Quality-of-life after total pancreatectomy: is it really that bad on long-term follow-up? J Gastrointest Surg 2005;9:1059-1066; discussion 1066-1067.

17 Jethwa P, Sodergren M, Lala A, Webber J, Buckels JA, Bramhall SR, Mirza DF: Diabetic control after total pancreatectomy. Dig Liver Dis 2006;38:415-419.

18 Casadei R, Monari F, Buscemi S, Laterza M, Ricci C, Rega D, D'Ambra M, Pezzilli R, Calculli L, Santini D, Minni F: Total pancreatectomy: indications, operative technique, and results: a single centre experience and review of literature. Updates Surg 2010;62:41-46.

19 Datta J, Lewis RS Jr, Strasberg SM, Hall BL, Allendorf JD, Beane JD, Behrman SW, Callery MP, Christein JD, Drebin JA, Epelboym I, He J, Pitt HA, Winslow E, Wolfgang C, Lee MK 4th, Vollmer CM Jr: Quantifying the burden of complications following total pancreatectomy using the postoperative morbidity index: a multi-institutional perspective. J Gastrointest Surg 2015;19:506-515.

20 Hartwig W, Gluth A, Hinz U, Bergmann F, Spronk PE, Hackert T, Werner J, Büchler MW: Total pancreatectomy for primary pancreatic neoplasms: renaissance of an unpopular operation. Ann Surg 2015;261:537-546. 
21 Heidt DG, Burant C, Simeone DM: Total pancreatectomy: indications, operative technique, and postoperative sequelae. J Gastrointest Surg 2007;11:209-216.

-22 Tai DS, Shen N, Szot GL, Posselt A, Feduska NJ, Habashy A, Clerkin B, Core E, Busuttil RW, Hines OJ, Reber HA, Lipshutz GS: Autologous islet transplantation with remote islet isolation after pancreas resection for chronic pancreatitis. JAMA Surg 2015;150: 118-124.

23 Crosby J, Bellin MD, Radosevich DM, Chinnakotla S, Dunn TB, Pruett TL, Freeman ML, Beilman GJ, Schwarzenberg SJ: Gastrointestinal symptoms before and after total pancreatectomy with islet autotransplantation: the role of pancreatic enzyme dosing and adherence. Pancreas 2015;44:453-458.

24 Georgiev G, Beltran Del Rio M, Gruessner A, Tiwari M, Cercone R, Delbridge M, Grigsby B, Gruessner R, Rilo H: Patient quality of life and pain improve after autologous islet transplantation (AIT) for treatment of chronic pancreatitis: 53 patient series at the University of Arizona. Pancreatology 2015;15:40-45.
25 Murphy MM, Knaus WJ 2nd, Ng SC, Hill JS, McPhee JT, Shah SA, Tseng JF: Total pancreatectomy: a national study. HPB (Oxford) 2009;11:476-482.

26 Tanaka M, Fernández-del Castillo C, Adsay V, Chari S, Falconi M, Jang JY, Kimura W, Levy P, Pitman MB, Schmidt CM, Shimizu M, Wolfgang CL, Yamaguchi K, Yamao K: International consensus guidelines 2012 for the management of IPMN and MCN of the pancreas. Pancreatology 2012;12:183-197.

27 Del Chiaro M, Verbeke C, Salvia R, Klöppel G, Werner J, McKay C, Friess H, Manfredi R, Van Cutsem E, Löhr M, Segersvärd R: European experts consensus statement on cystic tumours of the pancreas. Dig Liver Dis 2013;45:703-711.

-28 Takami H, Fujii T, Kanda M, Suenaga M, Yamamura K, Kodera Y: Preservation of the pyloric ring confers little benefit in patients undergoing total pancreatectomy. World J Surg 2014;38:1807-1813.
29 Fischer CP, Hong JC: Method of pyloric reconstruction and impact upon delayed gastric emptying and hospital stay after pylorus-preserving pancreaticoduodenectomy. J Gastrointest Surg 2006;10:215-219.

30 Choi SH, Hwang HK, Kang CM, Yoon CI, Lee WJ: Pylorus- and spleen-preserving total pancreatoduodenectomy with resection of both whole splenic vessels: feasibility and laparoscopic application to intraductal papillary mucin-producing tumors of the pancreas. Surg Endosc 2012;26:2072-2077.

31 Kim DH, Kang CM, Lee WJ: Laparoscopicassisted spleen-preserving and pylorus-preserving total pancreatectomy for main duct type intraductal papillary mucinous tumors of the pancreas: a case report. Surg Laparosc Endosc Percutan Tech 2011;21:e179-e182.

-32 Fortner JG: Regional resection of cancer of the pancreas: a new surgical approach. Surgery 1973;73:307-320.

33 Frigerio I, Girelli R, Giardino A, Regi P, Salvia $\mathrm{R}$, Bassi C: Short term chemotherapy followed by radiofrequency ablation in stage III pancreatic cancer: results from a single center. J Hepatobiliary Pancreat Sci 2013;20:574-577. 